B I O S C I E N C E

J O U R N A

\title{
PUNCTUAL MECHANICAL OSCILLATION IN MODULATION OF MUSCULAR TONUS IN CHILDREN WITH SPASTICITY
}

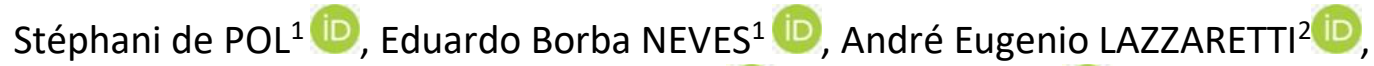 \\ Suhaila Mahmoud SMAILI ${ }^{3}$ (iD), Eddy KRUEGER ${ }^{4}$ ic
}

\footnotetext{
${ }^{1}$ Graduate Program of Biomedical Engineering, Federal Technological University of Paraná, Curitiba, Paraná, Brazil.

${ }^{2}$ Graduate Program in Electrical and Computer Engineering, Federal Technological University of Paraná, Curitiba, Paraná, Brazil.

${ }_{3}$ Master and Doctoral Program in Rehabilitation Sciences UEL-UNOPAR, Physiotherapy Department, State University of Londrina, Londrina, Paraná, Brazil.

${ }^{4}$ Neural Engineering and Rehabilitation Laboratory, Anatomy department, State University of Londrina, Paraná, Brazil.
}

Corresponding author:

Eduardo Borba Neves

Email: neveseb@gmail.com

How to cite: De POL, S., et al. Punctual mechanical oscillation in modulation of muscular tonus in children with spasticity. Bioscience Journal. 2021, 37, e37069. https://doi.org/10.14393/BJ-v37n0a2021-53574

\begin{abstract}
Spasticity is a motor condition present in 75 to $88 \%$ of children with Cerebral Palsy (CP). One form of treatment is called punctual mechanical oscillation (PO). The current study aimed to study different protocols for the application of PO and the magnitude of their effects. In total, 7children with medical diagnosis of CP and ICD (International Classification of Diseases) were included. The first intervention protocol $\left(\operatorname{Int}^{1}\right)$ consisted of the application of PO to the spastic muscle tendon and the second intervention protocol $\left(\operatorname{Int}^{2}\right)$ to the muscle belly ofthe spastic antagonist muscle. For evaluation, the Modified Ashworth Scale (MAS) was used, while simultaneously capturing the mechanomyography (MMG) signals. Data were

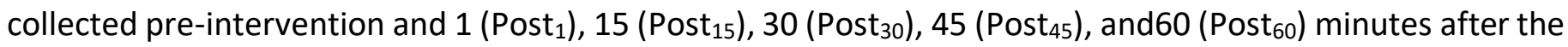
interventions. The MAS values (median \pm interquartile range) post intervention were statistically lower when compared to the pre values in the 2 protocols studied; in Int ${ }^{1}$ between Pre $(2 \pm 0)$ andPost $15(0 \pm 1.75)$, Post Po $_{30}$ $(0 \pm 1)$, Post $_{45}(1 \pm 1)$, and Post $60(1 \pm 1)$, and in Int ${ }^{2}$ only between Pre $(2 \pm 1)$ and Post $_{1}(0 \pm 1)$. The values found in the MMG in both its temporal and spectral domains did not follow a pattern $(p>0.05)$. The comparison between the protocols did not demonstrate statistical differences in any characteristics (MAS, MMG $\mathrm{MF}_{\text {, and }}$ $\left.M M G_{R M S}\right)$. However, PO was shown to be a therapeutic resource that modulated spasticity for up to 60 minutes after its application, and PO could contribute as a tool to aid the treatment of spasticity.
\end{abstract}

Keywords: Cerebral Pals. Mechanomyography. Modified Ashworth Scale. Muscle belly. Muscle tendon.

\section{Introduction}

Cerebral Palsy (CP) describes a group of disorders that compromise movement and posture, causing limitations in the functional activities of the individual. The incidence of CP in developed countries is approximately 2.0 to 2.5 per 1000 live births (Reddihough and Collins 2003; Rosenbaum et al. 2007), while in developing countries this rate can rise to 7 per 1000, it's believed that due to poor conditions of prenatal care and primary health care of pregnant women (Zanini et al. 2009). However, some current estimates indicate that $34-45 \%$ of CP cases may be due to genetic or epigenetic factors, and despite improvements in prenatal and perinatal care and advances in obstetric practices, the incidence of CP has changed little in recent decades (Maclennan et al. 2015; Fahey et al. 2017). 
It is estimated that 75 to $88 \%$ of patients present, as part of their clinical characteristic, alteration in muscle tone, defined as spasticity, which can cause secondary damage such as reduction in muscle length, deformities, and loss of function (Dias et al. 2015). On the other hand, this increase in muscle tone may contribute to joint stabilization, and assist in postural control, facilitating changes in decubitus and transfers. Thus, spasticity is a condition to be modulated and not completely eliminated (O'shea 2008; Brasil 2009).

The human body is known to be a biomechanical structure that has sensitivity to vibration (Balbinot 2001). Vibration refers to mechanical oscillations around a reference point, which are defined by the evaluated frequency and amplitude (Batista et al. 2007). Vibratory stimulation results in neurophysiological alterations through muscle and tendon mechanoreceptors, such as the muscle spindle (MS) and Golgi tendon organ (GTO) (Fallon and Macefield 2007).

Based on these neurophysiological characteristics, the protocol for the application of punctual mechanical oscillation (PO) on the spastic muscle tendon has been studied as a resource for the treatment of spasticity, in addition to the protocol for the application of PO on the muscle belly of the spastic antagonist muscle (krueger-beck et al. 2010). The application of these mechanical oscillations promotes normal patterns of motor activity as a result of modulation of motoneuron and corticospinal pathway excitability (De Moraes Silva et al. 2011).

However, there is still no consensus on the differences in results after application of punctual oscillation on the tendon and on the muscular belly. There is also no definition of the time of effectiveness in tone modulation after the application of the two OP techniques. Katusic et al. (2013), highlighted the importance of the existence of parameters for the application of punctual oscillation in the treatment of spasticity, as well as the duration effects of this intervention for patients (Katusic et al. 2013). Another limitation observed is the lack of studies on the effects of the use of punctual oscillation in children with spasticity since the majority of the current studies are in adult patients after stroke. In these cases, the results indicated that there was improvement in the functional capacity of chronic stroke patients (Caliandro et al. 2012) and a decrease in spasticity, with positive results up to 30 minutes after PO application (Noma et al. 2012). Therefore, it is worth investigating whether these results would be similar for children with CP.

Thus, this study aims to verify the effectiveness of punctual mechanical oscillation in modulating spasticity in children with cerebral palsy using two application protocols (as well as to evaluate if there is superiority between them) and, additionally, to verify the modulation permanence time.

As this is an experimental work, the hypothesis of the study was that the two protocols of application of mechanical punctual oscillation would modulate spasticity, that the application of PO on the spastic muscle tendon would be more effective than application on the muscle belly of the spastic antagonist muscle(since when applied to the tendon, there may be the illusory sensation of movement, causing the inverse vibration tonic reflex to occur, as the GTO perceives the high tension that the muscle fibers suffer, and thus, as a form of protection, spasticity modulation occurs more effectively), and there would be an effect on tone modulation for up to $60 \mathrm{~min}$ after application.

\section{Material and Methods}

\section{Participants}

The project developed for this research was carried out in accordance with Resolution 466/2012 of the National Health Council and obtained approval from the Ethics and Research Committee of the Universidade Tecnológica Federal do Paraná - UTFPR under opinion number 1.859.869.

This is an experimental analytical case series study. Seven children of both sexes were selected from patients treated at the Institute of Health and Rehabilitation, with the headquarters located in Curitiba-PR and branches in Ponta Grossa-PR, Clinic Neuro Concept, Curitiba-PR and the Municipal Educational Center of specialized care Professor Lindamir Terezinha Ferreira Ribeiro - CEMAE, Campo Largo - PR. Patients with a proven medical diagnosis of cerebral palsy were included (ICD 80 - International Classification of Diseases), aged between 3 and 12 years, who presented spasticity greater than grade 1 in the biceps brachii muscle (BB), according to the Modified Ashworth Scale (MAS), and whose caregivers consented and signed the consent form. Patients who presented any dermatitis or skin lesions in the area of placement of the double- 
sided tape that adheres the mechanomyography sensor (MMG) to the patient and who presented any rejection of the stimulus from the punctual oscillation were excluded.

\section{Instrumentation}

The present study obtained results through the Modified Ashworth Scale (MAS) and Mechanomyography signals. For the collections of the MMG, 4-channel EMGSystem Ltda. equipment was used, with a triaxial accelerometer. The skin was prepared with 70ㅇ alcohol asepsis, and the sensor was positioned over the distal third of the BB muscle for both evaluation protocols.

For the MAS evaluation, the evaluator performed a stretching reflex on the elbow joint until it reached 0 o extension, or the participant's range of motion. Throughout the range of motion, MMG signals were captured as shown in Figure 1.

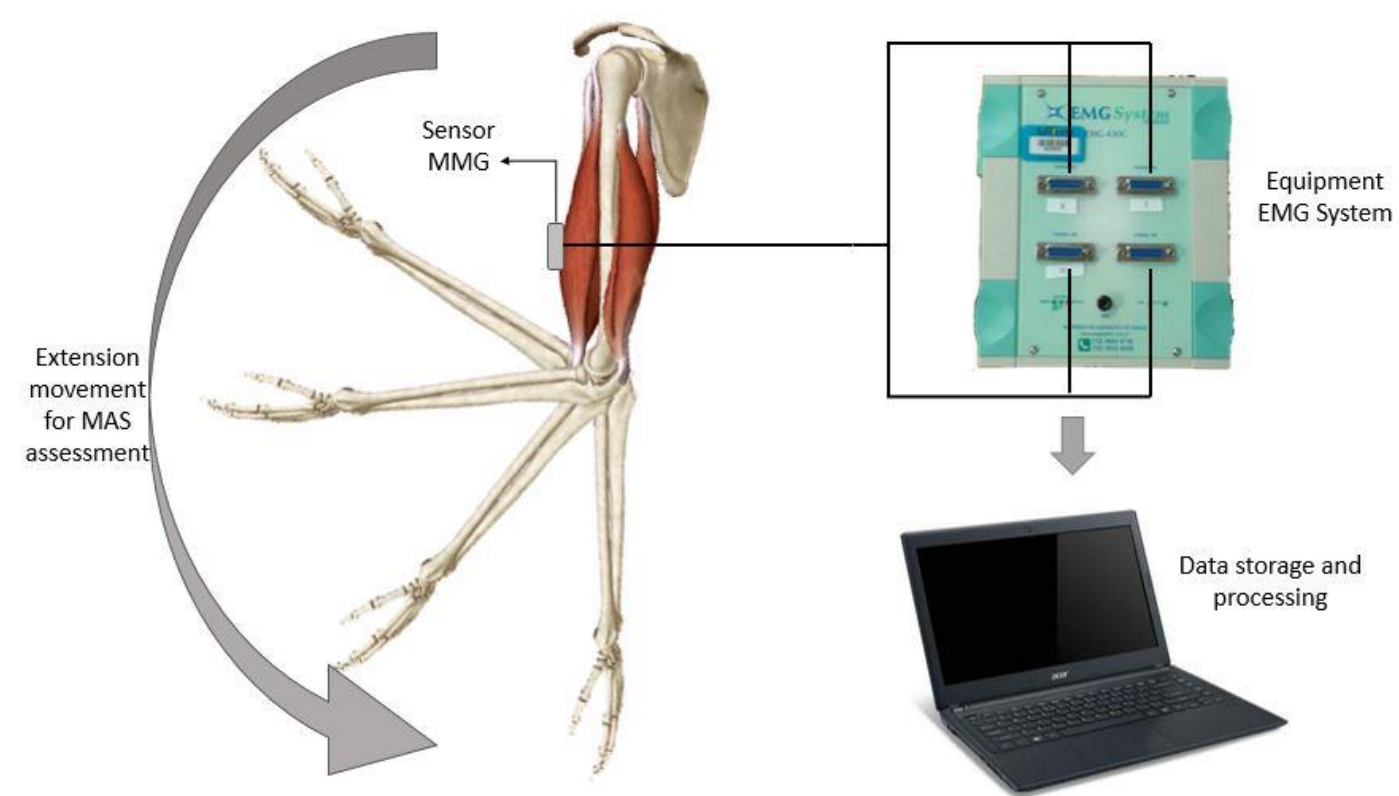

Figure 1. Diagram of the evaluation protocol, Mechanomyography (MMG) signal capture simultaneously with the modified Ashworth Scale evaluation (MAS).

\section{Experimental protocol}

Two interventions were performed in all participants, with a period of 7 to 15 days between the first and the second interventions, with the objective of evaluating two different methods of applying oscillation in the modulation of BB muscle spasticity.

The first intervention protocol $\left(\mathrm{Int}^{1}\right)$ was punctual oscillation (PO) on the BB muscle tendon for 15 minutes, as shown in Figure 2. The second intervention protocol $\left(\operatorname{lnt}^{2}\right)$ was performed on the muscle belly $(\mathrm{MB})$ of the spastic antagonist muscle, in this case, on the MB of the triceps brachii (TB) as shown in Figure 3 , respecting the same $\mathrm{PO}$ application time of 15 minutes. 


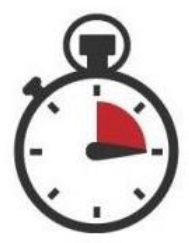

15 Minutes

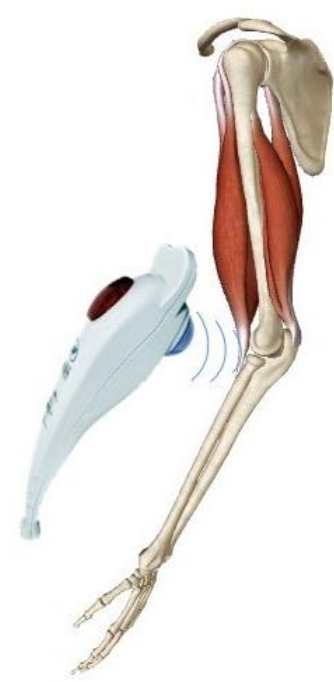

Figure 2. Application of Punctual Oscillation on the tendon of the biceps brachii spastic muscle.
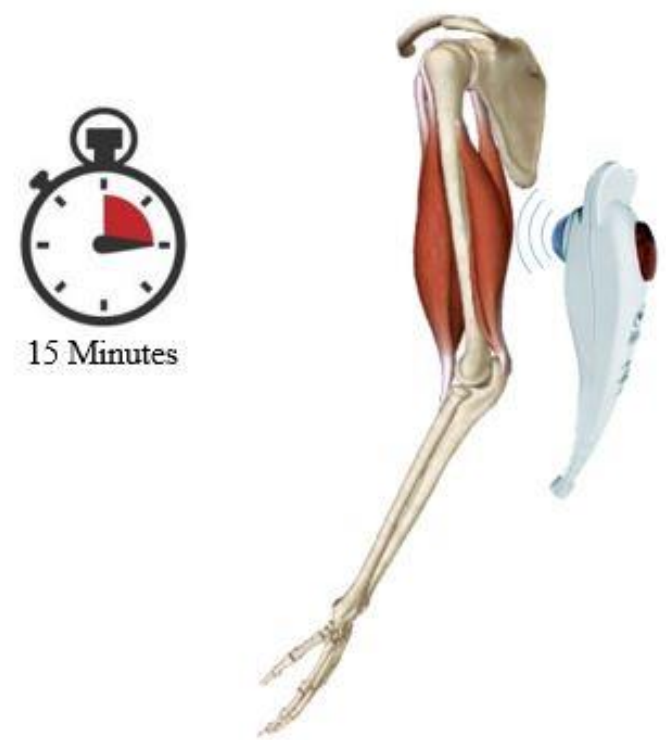

Figure 3. Application of Punctual Oscillation on the muscle belly of the triceps brachii, spastic antagonist muscle.

During the two interventions, the participants remained lying comfortably on a flat surface, and at the time of collection they flexed their shoulder and elbow at 45을 110 , respectively. The same form of application protocol was respected in both evaluations, except for the PO intervention site, as mentioned above.

The evaluations for both protocols consisted of the Ashworth Modified Scale evaluation of spasticity ( 0 without increase in muscle tone, 4 joint stiffness), while simultaneously capturing MMG signals before the application of punctual oscillation, called pre-intervention (Pre). After the pre-assessment, the intervention was performed with the punctual mechanical oscillation, using a massager from the company Relaxmedic $^{\circledR}$, model Infratch Hammer, with oscillation frequency of $45 \mathrm{~Hz}$ and amplitude of $0.5 \mathrm{~mm}$. The PO

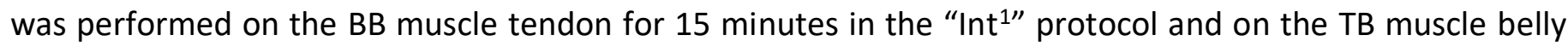
for 15 minutes in the "Int ${ }^{2 "}$ protocol.

After the end of the PO application, the values of MAS and MMG were recorded at the following moments: 1 minute (Post 1 ), 15 minutes (Post ${ }_{15}$ ), 30 minutes (Post ${ }_{30}$ ), 45 minutes (Post 45 ), and 60 minutes (Post60) after the intervention. 


\section{Processing}

For the processing and analysis of the data, the program MatLab ${ }^{\circledR}$ (MathWorks, version R2012b) was used. The digital filter chosen for the MMG signal was Butterworth, third order, with a 5-50 Hz bandwidth. The axis perpendicular to the muscle fibers ( $Z$ axis) was used for processing in the temporal domain, by the root mean square (Root Mean Square- $M M_{\mathrm{RMS}}$ ), and in the spectral, by the median of the frequency (MMG $\mathrm{MF}_{\mathrm{F}}$, which was processed by Discrete Fourier Transform.

\section{Statistical analysis}

In addition to the descriptive analysis using boxplot graphs, a statistical test was applied to corroborate the significance between the moments (Pre, Post 1, Post $_{15}$, Post 30, Post $_{45}$, and Post 60 ) and between the application techniques (muscle belly or muscle tendon). The statistical analysis was performed with custom-written MatLab ${ }^{\otimes}$ (MathWorks, Inc.) software, version R2015a. Because of the data skew, the Friedman test (related to non-parametric ANOVA) was computed for the data ( $p$ value $\leq 0.05$ ) in order to evaluate if the protocol moments were different among them. Considering statistical significance, for Post hoc analysis, the Bonferroni adjustment with P-value waschosen according to multiple comparisons; in this sense, differences were considered significant for $p \leq 0.0083$. The Wilcoxon test was applied between the techniques (muscle belly or muscle tendon) for each moment $(p \leq 0.05)$. Effect size $(d)$ was calculated (Cohen 2013) for significant data. The values for interpretation of effect size were: small ( $d=0.10-0.29)$, medium $(d=0.30-0.49)$, and large ( $d=>0.50)$ (Cohen 1988).The data found in this study are described as median \pm interquartile range.

\section{Results}

Seven children with a clinical diagnosis of Cerebral Palsy, with spasticity in the biceps brachii muscle participated in this study; one child with a clinical presentation of hemiparesis (spastic unilateral) and six with quadriparesis (spastic bilateral), ranging from 5 to 7 years of age ( 2 of 5 years, 3 of 6 years, and 3 of 7 years) and with varying levels of motor impairment, specified in Table 1 by the GMFCS (Gross Motor Function Classification System) and MACS (The Manual Ability Classification System) (Eliasson et al. 2006; Palisano et al. 2007).

Table 1. Description of the characteristics of the individuals analyzed.

\begin{tabular}{cccccc}
\hline & Age (years) & Sex & Topographic Diagnosis & GMFCS & MACS \\
\hline Individual 1 & 6 & Masculine & Quadriparesis & V & V \\
Individual 2 & 5 & Masculine & Quadriparesis & IV & V \\
Individual 3 & 6 & Masculine & Quadriparesis & V & V \\
Individual 4 & 7 & Masculine & Quadriparesis & V & V \\
Individual 5 & 7 & Feminine & Hemiparesis & I & II \\
Individual 6 & 5 & Feminine & Quadriparesis & V & IV \\
Individual 7 & 7 & Masculine & Quadriparesis & V & V \\
\hline
\end{tabular}

Due to the non-parametric distribution of the data collected, we opted for the analysis of the medians \pm interquartile range (Q3 value - Q1 value), of the MAS and MMG values in both their temporal and spectral domains, found in Int $^{1}$ and Int $^{2}$.

In Int ${ }^{1}$, represented by the letter (A) in Figure 4, the value found by the MAS in the pre-intervention was $2 \pm 0$. Soon after the intervention on the muscle tendon, at Post ${ }_{1}$, this reduced to $1 \pm 1.75$. AtPost ${ }_{15}$ the value decreased to $0 \pm 1.75$ and at Post 30 to $0 \pm 1$. At Post 45 andPost 60 the value returned to $1 \pm 1$.

In $\mathrm{Int}^{2}$, represented by the letter (B) in Figure 4, the value found by the MASPre-intervention was $2 \pm$ 1. At Post 1 , the median value decreased to $0 \pm 1$. At Post ${ }_{15}$ the value rose to $1 \pm 1.5$. At Post 30 the median value remained at $1 \pm 1$, at Post $451 \pm 0.75$, and at Post $601 \pm 0$. 

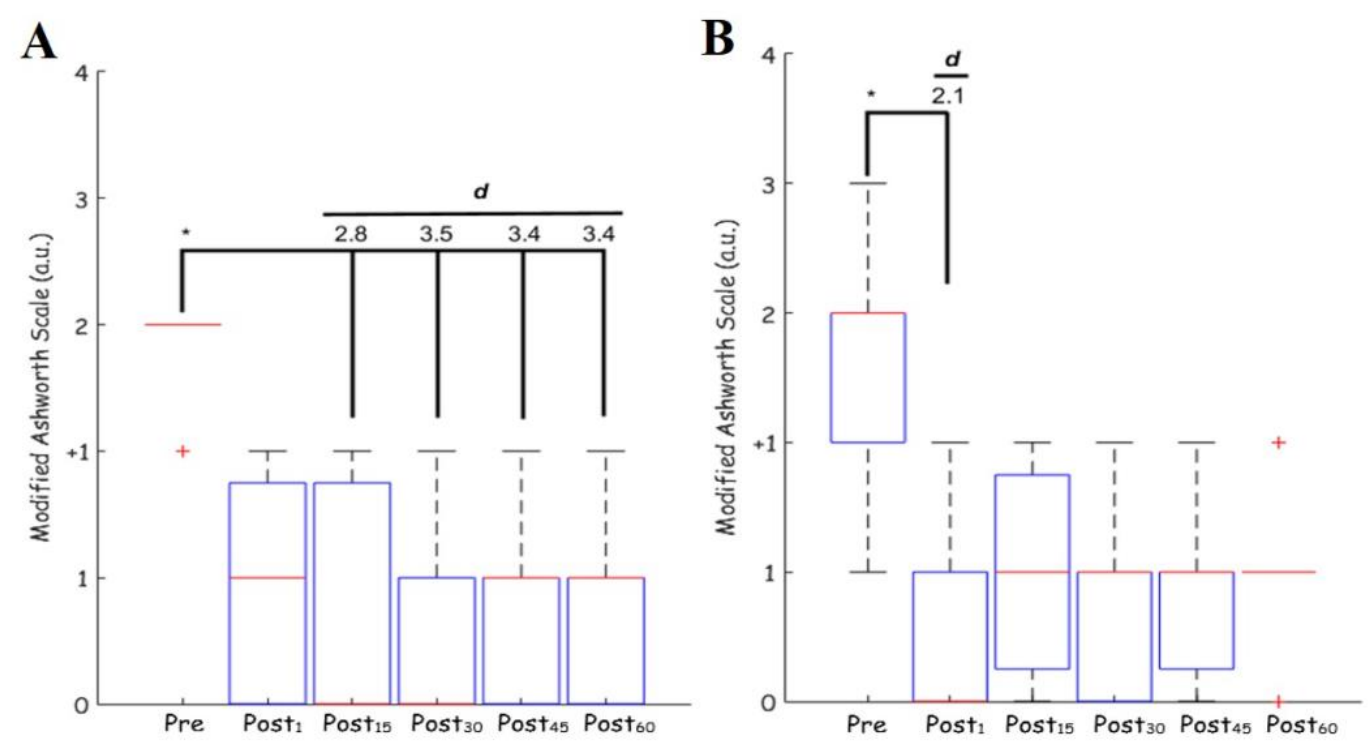

Figure 4. A - modified Ashworth Scale of muscle tendon; B - modified Ashworth Scale of muscle belly. $*=p \leq 0.0083 . d=$ effect size. Red cross = outliers.

Figure 5 presents the variations found in the median frequency $M M G$ ( $M M G_{M F}$ ) in the collection in $\operatorname{lnt}^{1}(A)$ and $1 \operatorname{lnt}^{2}(B)$ for the 7 individuals. The median of the values found in $\operatorname{lnt}^{1}(A)$ in the Pre-intervention was $14 \pm 16.75 \mathrm{~Hz}$, rising at Post ${ }_{1}$ to $16 \pm 5 \mathrm{~Hz}$ and decreasing at Post ${ }_{15}$ to $14 \pm 5 \mathrm{~Hz}$. AtPost 30 the value rose again to $16 \pm 3.5 \mathrm{~Hz}$, at Post 45 the value decreased to $13 \pm 7.25 \mathrm{~Hz}$, and at Post 60 the value rose again to 17 $\pm 4.75 \mathrm{~Hz}$.

Still in Figure 5, now for the Int $^{2}$, represented by the letter (B), a Pre value of $15 \pm 3 \mathrm{~Hz}$ was obtained, and at Post ${ }_{1}$ the value remained at $15 \pm 8 \mathrm{~Hz}$. Soon after, at Post 15 the value rose to $17 \pm 7.5 \mathrm{~Hz}$, at Post 30 decreased again to $15 \pm 1.75 \mathrm{~Hz}$, at Post 45 rose again to $21 \pm 11 \mathrm{~Hz}$, and at Post 60 the value decreased to 18 $\pm 8.5 \mathrm{~Hz}$.
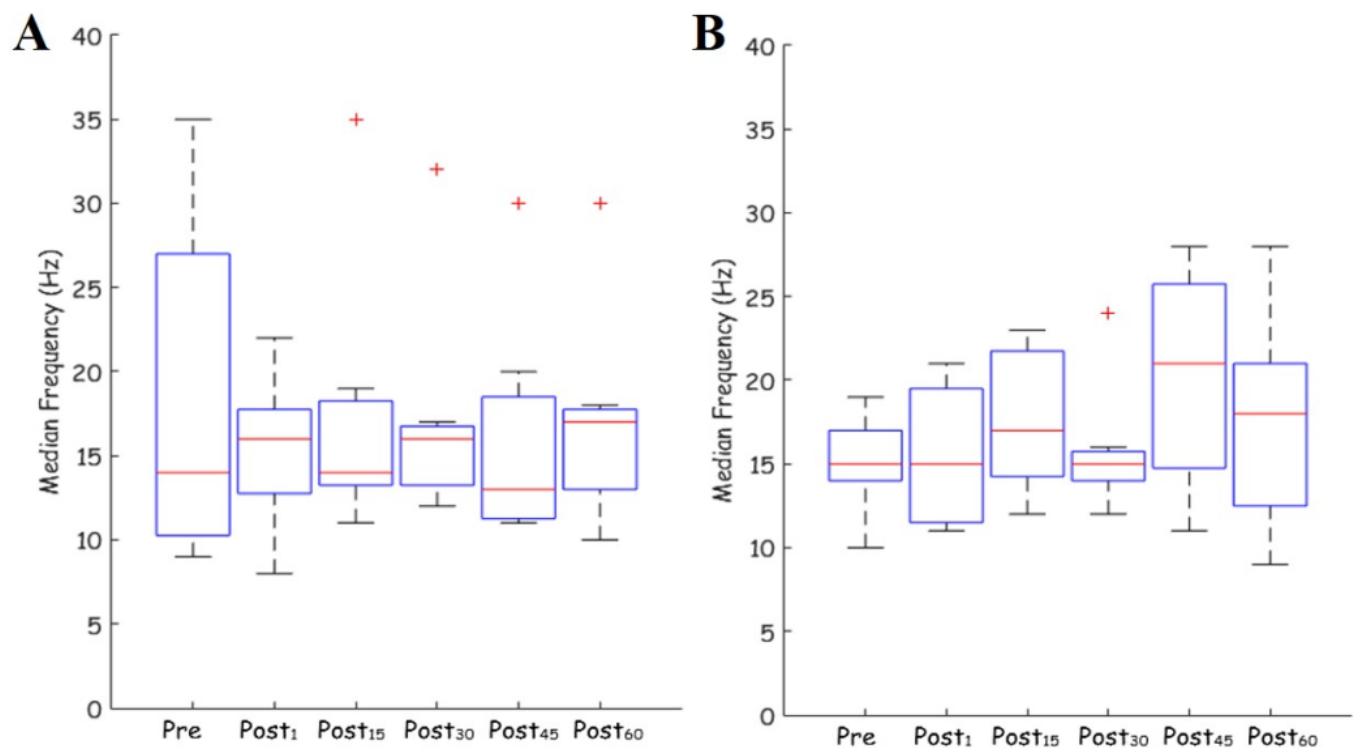

Figure 5. A - mechanomyography median frequency of muscle tendon; B - mechanomyography median frequency of muscle belly. Red cross = outliers.

Figure 6 presents the values of MMG in the temporal domain ( $M M G_{R M S}$ ) of the 7 analyzed individuals, where $G$ is the acceleration of gravity, with the magnitude given in $\left(\mathrm{ms}^{-2}\right)$; Int ${ }^{1}$ is represented by $(A)$ and $I n t^{2}$ by (B). For $\mathrm{Int}^{1}$, the values found at Pre were $0.049 \pm 0.031 \mathrm{G}_{\mathrm{RMS}}$. The value decreased atPost ${ }_{1}$ to $0.041 \pm 0.032$ $\mathrm{G}_{\mathrm{RMS}}$, atPost ${ }_{15}$ to $0.035 \pm 0.038 \mathrm{G}_{\mathrm{RMS}}$, at Post 30 to $0.034 \pm 0.063 \mathrm{G}_{\mathrm{RMS}}$, at Post ${ }_{45}$ to $0.033 \pm 0.076 \mathrm{G}_{\mathrm{RMS}}$, and finally, at Post 60 to0.027 $\pm 0.024 \mathrm{G}_{\mathrm{RMS}}$. 
For $\mathrm{Int}^{2}$, the $\mathrm{MMG}_{\mathrm{RMS}}$ value found for Pre was $0.035 \pm 0.024 \mathrm{G}$. At Post ${ }_{1}$ the value rose to0.047 \pm $0.025 \mathrm{G}$, at Post ${ }_{15}$ decreased to0.031 $\pm 0.017 \mathrm{G}$, rising again at Post ${ }_{30}$ to $0.035 \pm 0.030 \mathrm{G}$, reducing at Post $_{45}$ to $0.031 \pm 0.017 \mathrm{G}$, and at Post 60 rising to $0.042 \pm 0.026 \mathrm{G}$.

$\mathbf{A}$

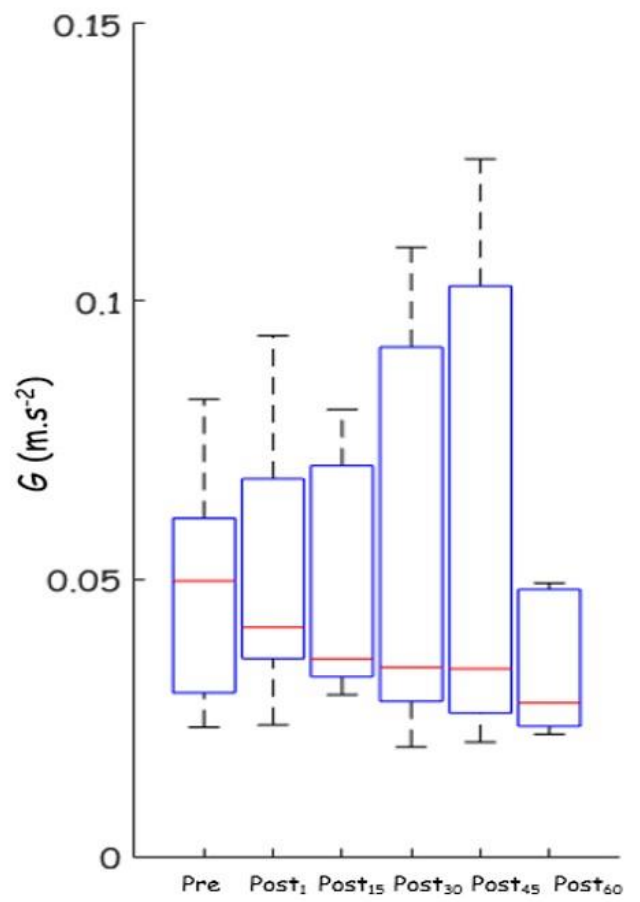

B

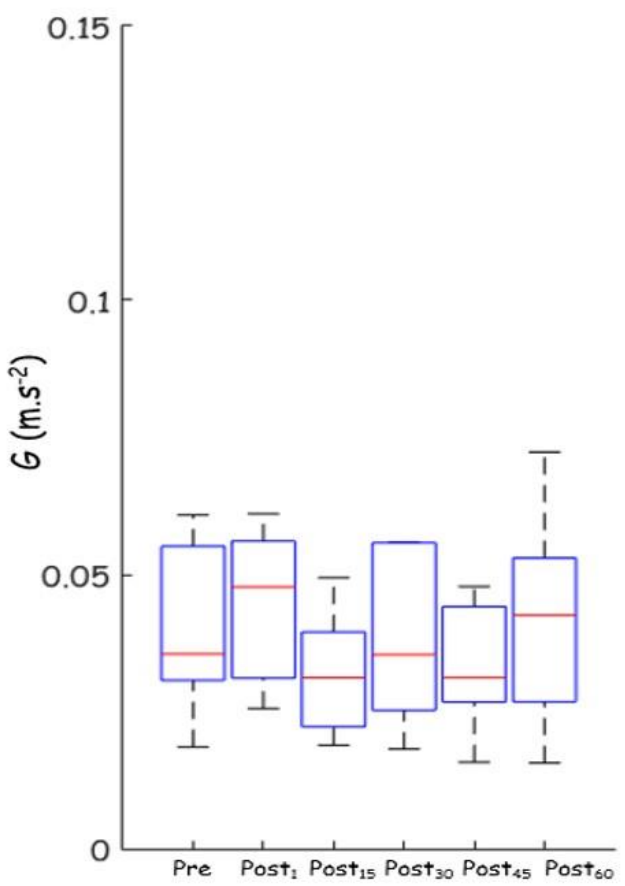

Figure 6. A - mechanomyography root mean square of muscle tendon; B - mechanomyography root mean square of muscle belly. Red cross = outliers.

The comparison between techniques (muscle tendon or muscle belly) did not demonstrate statistical significance in any features (MAS, $M M_{M F}$, and $M M G_{R M S}$ ).

\section{Discussion}

Through the data analysis, the Wilcoxon test, applied to both protocols, $\operatorname{lnt}^{1}$ (muscle tendon) and $\operatorname{lnt}^{2}$ (muscle belly), at each moment analyzed, demonstrated a result of $p \geq 0.05$, i.e., both techniques were similar when evaluated in terms of results and it cannot be stated that one protocol is superior to the other.

The effect size (d) of the techniques was calculated using the Cohen test (Cohen 2013) and the data were significant, with $p \geq 0.8$, indicating that both techniques presented satisfactory results for the analyzed question, that is, for the modulation of spasticity in children with $\mathrm{CP}$, considering the measurement by means of MAS.

In the present study, the 7 individuals analyzed demonstrated a reduction in the values found by the MAS in both forms of intervention. However, in the Int $^{1}$ protocol there was statistical significance at the post-intervention moments of $15,30,45$, and 60 minutes when compared to the median value Preintervention, while in the Int $^{2}$ protocol, there was statistical significance only at the Post ${ }_{1}$ moment when compared to the Pre-intervention.

In a study with a similar protocol, performed Katusic et al. (2013), 89 children aged 4 to 6 years diagnosed with CP underwent 2-weekly interventions for 12 weeks with a $40 \mathrm{~Hz}$ frequency vibration for 20 minutes in each session. The authors observed a reduction in MAS and improved motor performance assessed by the gross motor function scale (GMFM-88), when compared to a control group that did not undergo the intervention with punctual oscillation. In the same study, the authors highlighted the importance of the existence of parameters of the application of punctual oscillation in the treatment of spasticity, as well as the effectiveness of the duration of this intervention for the patient and the research aimed at this public (Katusic et al. 2013). 
The values found in the two MMG domains varied and there was no correlation with the values found by the MAS. This is different from the findings in a study conducted in 2016, which sought to correlate the levels of MAS spasticity with MMG signals. The reported results showed correlation of the values found by the MAS in the temporal domain, while in the spectral domain there was a lower correlation (Santos 2016).

A study by Conrad et al. (2011), used the application of vibration on the wrist flexor spastic tendon in 10 post-stroke patients, and observed reduced muscle activity and improved movement control (Conrad et al. 2011). The same authors, who in 1968 described vibratory tonic reflex when spinal PO was performed (Hagbarth and Eklund 1968), two years earlier in 1966 had already described the benefits of PO on the spastic tendon, describing the improvement in motor control and also that the effects of spasticity modulation are maintained even after ceasing the PO (Hagbarth and Eklund 1966).

Although there was no statistical correlation between MAS and MMG, the application of PO on the tendon evaluated by the MMG in its temporal domain showed a decline when comparing the pre value with the post-application values, since in the Pre-application value we found a median of $0.049 \pm 0.031 \mathrm{G}_{\mathrm{RM}}$ to $0.027 \pm 0.024 G_{R M s}$ at $P_{0 s t}{ }_{60}$, however, when statistically evaluated there was no difference between these moments. Different fromlnt ${ }^{2}$, in Int $^{1}$ however, there was a decline in the MAS value after application at Post ${ }_{1}$, however not fully modulating muscle tone, but then further declining to 0 at Post ${ }_{15}$ and Post 30 and rising back to 1 at Post 45 and Post 60 . Despite this return to a value of 1 through the MAS, the median value Post $_{60}$ was still lower than the median value of Pre.

When we observed the evaluation by the MAS in the Int $^{1}$ protocol, the size of the effect of Post moments in relation to Pre-intervention, we observed that at Post ${ }_{1}$ there was no statistical difference, indicating that the modulation occurs more effectively 15 minutes after intervention, remaining up to 60 minutes after its application.

A study of great relevance showed that besides the modulation of spasticity, there are other associated gains. That study evaluated patients with multiple sclerosis who had spasticity in lower limbs, and identified that after the application of vibration, in addition to the reduction in spasticity, individuals also presented pain reduction assessed by the ID-Pan pain questionnaire and improvement in quality of life assessed by the FS-36 questionnaire. This indicates that PO is a safe tool, which, by producing spasticity modulation benefits the individual who received the application in several ways (Camerota et al. 2017).

The result of this modulation is due to what is called the inverse vibratory tonic reflex (IVTR), since when the oscillation on the spastic tendon was performed there is an illusory sensation of movement and the GTO perceives the high tension suffered by the muscle fiber, in order to minimize this effect the agonist muscle is inhibited by the internuncial cell (Golgi type II) present in the spinal cord gray matter and, simultaneously, this fact is accompanied by the activation of the antagonist muscle (Gillies et al. 1969; Ortolan et al. 2017). Thus, there is better synergy during the flexion-extension movement of the elbow, as described in this study and evidenced by MAS.

Studies such as by Hagbart et al. justify the reasons why vibration on the muscle belly of the spastic antagonist muscle modulates spasticity, as when there is PO on the muscle belly there is a reflex response of a sustained contraction of the vibrated muscle and a simultaneous relaxation of its antagonist, this response being called Vibrating Tonic Reflex (VTR) (Hagbarth and Eklund 1968). The same occurred when in the present study the PO was applied to the triceps brachii muscle spindle, as this application caused its sustained contraction, consequently reducing the muscle tone of the biceps brachii, thus helping to improve limb mobility.

As in the present study, the effectiveness of PO on the MV of the spastic antagonist muscle could be evidenced, since the application of PO on the triceps brachii increased the motor excitability of this muscle and reduced the motor excitability of the biceps brachii, modulating the spasticity and making the individual movement more functional.

A study that performed the application of $\mathrm{PO}$ in children with $\mathrm{CP}$ showed that the application on the muscle belly of the spastic muscle (using the calf muscles), improved the ADM evaluated by the digital goniometer, also suggesting that there was modulation in spasticity and improved joint movement (Yun et al. 2015).

Improved synergy in elbow flexion-extension motion can be evidenced by a reduction in MAS through the median value at Pre of 2compared to 1 at Post 60 minutes, thus indicating that the PO on the MB of the 
spastic antagonist also has effects on modulation of spasticity in children. Statistical analysis of the effect size showed a significant difference only when comparing the Pre to Post1 moments, indicating an improvement in spasticity, with an acute effect of PO application on the muscle belly of the spastic. Similarly to Int $^{1}$, there was no statistical correlation between MAS and MMG.

Still responding to the objectives of this study, it was possible to verify that in both forms of PO application there were reductions in the values found by MAS, since it was observed that the spasticity up to Post 60 presented values lower than at Pre-intervention, indicating that both techniques of PO modulate spasticity for up to one hour after application.

Corroborating this fact, the study by Burke et al. (1972) in patients with spasticity, reported improved spasticity for up to two hours after ceasing vibration, and that the patient limbs were more relaxed and free of spasms during this period (Burke et al. 1972). A recent study by Li et al. (2019) verified by electroencephalogram examination that application of PO to the muscles of the upper limbs activates the bilateral primary somatosensory cortex (Li et al. 2019).

In 2012, a study by Noma et al. (2012) investigated the direct application of vibratory stimuli to inhibit upper limb spasticity in 36 post-stroke patients, performed for 5 minutes and evaluated by MAS and EMG 1 min and 30 min after application. Three vibrators were positioned, one on the metacarpophalangeal joint, and another two on the biceps brachii MB, that is, on the spastic muscles. Even with a different application from the protocol applied in the present study, the authors stated that continuous vibratory stimulation for a few minutes applied directly to the spastic muscle produces an intense initial contraction followed by decreased spasticity, with positive results up to 30 minutes. At the end of the study the authors suggested further investigations of the time of the effect in reducing spasticity (Noma et al. 2012); which, as previously stated, was performed in the present study, indicating the effectiveness of spasticity modulation for up to 60 min after application.

This study has some limitations; the sample number was non-probabilistic (higher chances of occurrence of type II error), the age of the participants does not cover all age groups, and due to the number of participants, there was no large demographic coverage. Patients in the sample were required to have a proven medical report of ICD10 G80 Cerebral Palsy (G80.0 Spastic quadriplegic cerebral palsy, G80.1 Spastic diplegic cerebral palsy, G80.2 Spastic hemiplegic cerebral palsy, and G80.8 Other forms of cerebral palsy), and this was a limiting factor, since many patients who underwent screening had no proven medical diagnosis and could not be included in the sample of the present study. Another point to consider is that in this study there was no blinding.

\section{Conclusions}

The objective of this study was to verify the effectiveness of punctual mechanical oscillation in the modulation of spasticity, to verify the modulation permanence time, as well as to compare the two application protocols using the Modified Ashworth Scale and Mechanomyography. From the results, it can be concluded that:

a) The use of punctual oscillation in both application forms presented satisfactory results in the modulation of spasticity when analyzing the values found by the Modified Ashworth Scale. However, the values found by the MMG did not follow a pattern when compared to the MAS, due to the possible associated limb movement when performing the stretching reflex in the MAS evaluation, as the signals of this movement were also possibly captured by the accelerometer. The statistical analyzes of the comparison between the protocols (muscle tendon or muscle belly) did not show statistical significance in any characteristics (MAS, MMG protocol on the tendon would be more effective than on the muscle belly.

b) Both intervention protocols with PO, when analyzed by MAS, were effective even 60 minutes after the intervention, corroborating the primary hypothesis raised by the researchers.

Applications with PO have been shown to be effective in modulating spasticity, as peripheral stimuli induced by PO improve the control of motoneuron excitability and induce neural pathway remodeling, thus contributing to central nervous system plasticity. The improved muscle synergy achieved through PO also helps these spastic children to gain better movement control and thus learn motor function and ranges, which contributes to their normal motor development. Although the limitations cited by this study should 
be addressed and larger samples are needed to confirm our results, we can suggest that PO can be used to increase the functional outcomes obtained to improve the spasticity and function in patients receiving this intervention.

Authors' Contributions: De POL, S.: conception and design, acquisition of data, analysis and interpretation of data, drafting the article; KRUEGER, E.: conception and design, acquisition of data, analysis and interpretation of data, drafting the article; NEVES, E.B.: conception and design, analysis and interpretation of data, drafting the article; LAZZARETTI, A.E.: analysis and interpretation of data, drafting the article; SMAILI, S.M.: analysis and interpretation of data, drafting the article. All authors have read and approved the final version of the manuscript.

Conflicts of Interest: The authors declare no conflicts of interest.

Ethics Approval: Approved by Research Ethics Committee of Federal Technological University of Paraná. Number: 1.859.869.

Acknowledgments: The authors would like to thank the funding for the realization of this study provided by the Brazilian agencies CAPES (Coordenação de Aperfeiçoamento de Pessoal de Nível Superior - Brasil), Finance Code 001, and CNPq (Conselho Nacional de Desenvolvimento Científico e Tecnológico - Brasil). We would also like to say thanks to the Ergonomics Laboratory (LAERG) of UTFPR, for the loan of the mechanomyography equipment.

\section{References}

BALBINOT, A. Caracterização dos níveis de vibração em motoristas de ônibus: um enfoque no conforto e na saúde. 2001. Available from: https://www.lume.ufrgs.br/handle/10183/248

BATISTA, M.A., et al. Efeitos do treinamento com plataformas vibratórias. Revista Brasileira de Ciência e Movimento. 2007, 15(3), 103-114. http://dx.doi.org/10.31501/rbcm.v15i3

BRASIL. Ministério da Saúde. Protocolo clínico e diretrizes terapêuticas espasticidade. 2009. Available from: http://portalarquivos.saude.gov.br/images/pdf/2017/julho/03/PCDT-Espasticidade_29_05_2017.pdf.

BURKE, D., ANDREWS, C.J. and LANCE, J.W. Tonic vibration reflex in spasticity, Parkinson's disease, and normal subjects. Journal of Neurology, Neurosurgery \& Psychiatry. 1972, 35(4), 477-486. http://dx.doi.org/10.1136/jnnp.35.4.477

CALIANDRO, P., et al. Focal muscle vibration in the treatment of upper limb spasticity: a pilot randomized controlled trial in patients with chronic stroke. Archives of physical medicine and rehabilitation. 2012, 93(9), 1656-1661. https://doi.org/10.1016/j.apmr.2012.04.002

CAMEROTA, F., et al. Focal muscle vibration, an effective rehabilitative approach in severe gait impairment due to multiple sclerosis. Journal of the neurological sciences. 2017, 372, 33-39. https://doi.org/10.1016/i.jns.2016.11.025

COHEN, J. Statistical power analysis for the behavioral sciences. Abingdon: Routledge. 1988, pp. 567.

COHEN, J. Statistical power analysis for the behavioral sciences. Academic press Routledge: Nova York. Revised Edition. 2013.

CONRAD, M.O., SCHEIDT, R.A. and SCHMIT, B.D. Effects of wrist tendon vibration on arm tracking in people poststroke. Journal of neurophysiology. 2011, 106(3), 1480-1488. https://doi.org/10.1152/jn.00404.2010

DE MORAES SILVA, J., LIMA, M.O. and DE PAULA JÚNIOR, A.R. Efeito agudo da estimulação vibratória em hemiparéticos espásticos pósacidente vascular encefálico. Research on Biomedical Engineering. 2011, 27(4), 224-230. http://dx.doi.org/10.4322/rbeb.2011.018

DIAS, C.P., et al. Paralisia cerebral em Pediatria. Pediatria moderna. 2015. 51(6), 224-229.

ELIASSON, A.C., et al. The Manual Ability Classification System (MACS) for children with cerebral palsy: scale development and evidence of validity and reliability. Developmental medicine \& child neurology. 2006, 48(7), 549-554. https://doi.org/10.1017/S0012162206001162

FAHEY, M.C., et al. The genetic basis of cerebral palsy. Developmental Medicine \& Child Neurology. 2017, 59(5), $462-469$.

https://doi.org/10.1111/dmcn.13363

FALLON, J.B. and MACEFIELD, V.G. Vibration sensitivity of human muscle spindles and Golgi tendon organs. Muscle \& nerve. 2007, 36(1), 21-29. https://doi.org/10.1002/mus.20796

GILLIES, J., LANCE, J., NEILSON, P. and TASSINARI, C. Presynaptic inhibition of the monosynaptic reflex by vibration. The Journal of physiology. 1969, 205(2), 329-339. https://doi.org/10.1113/jphysiol.1969.sp008968

HAGBARTH, K.-E. and EKLUND, G. The effects of muscle vibration in spasticity, rigidity, and cerebellar disorders. Journal of neurology, neurosurgery, and psychiatry. 1968, 31(3), 207. http://dx.doi.org/10.1136/jnnp.31.3.207

HAGBARTH, K. and EKLUND, G. Tonic vibration reflexes (TVR) in spasticity. Brain research. 1966, 2(2), 201. https://doi.org/10.1016/0014$\underline{4886(66) 90088-4}$

KATUSIC, A., ALIMOVIC, S. and MEJASKI-BOSNJAK, V. The effect of vibration therapy on spasticity and motor function in children with cerebral palsy: a randomized controlled trial. NeuroRehabilitation. 2013, 32(1), 1-8. https://doi.org/10.3233/NRE-130817

KRUEGER-BECK, E., NOGUEIRA-NETO, G.N. and NOHAMA, P. Estímulo vibracional na espasticidade-uma perspectiva de tratamento. Revista Neurociências. 2010, 18(4), 523-530. https://doi.org/10.4181/RNC.2010.ip02.7p 
LI, W., LI, C., XU, Q. and JI, L. Effects of Focal Vibration over Upper Limb Muscles on the Activation of Sensorimotor Cortex Network: An EEG Study. Journal of Healthcare Engineering. 2019, 2019, 2040-2295. https://doi.org/10.1155/2019/9167028

MACLENNAN, A.H., THOMPSON, S.C. and GECZ, J. Cerebral palsy: causes, pathways, and the role of genetic variants. American Journal of Obstetrics \& Gynecology. 2015, 213(6), 779-788. https://doi.org/10.1016/j.ajog.2015.05.034

NOMA, T., et al. Anti-spastic effects of the direct application of vibratory stimuli to the spastic muscles of hemiplegic limbs in post-stroke patients: a proof-of-principle study. Journal of rehabilitation medicine. 2012, 44(4), 325-330. https://doi.org/10.2340/16501977-0946

O'SHEA, T.M. Diagnosis, treatment, and prevention of cerebral palsy in near-term/term infants. Clinical obstetrics and gynecology. 2008, 51(4), 816. https://doi.org/10.1097/GRF.0b013e3181870ba7

ORTOLAN, R.L., et al. Tratamento de terapia vibratória em pacientes com espasticidade. Fisioterapia em Movimento. 2017, 18(1), 67-74.

PALISANO, R., et al. GMFCS - E\&R: Gross Motor Function Classification System - Expanded \& Revised. 2007. Available from: https://canchild.ca/en/resources/42-gross-motor-function-classification-system-expanded-revised-gmfcs-e-r

REDDIHOUGH, D.S. and COLLINS, K.J. The epidemiology and causes of cerebral palsy. Australian Journal of physiotherapy. $2003,49(1), 7-12$. https://doi.org/10.1016/S0004-9514(14)60183-5

ROSENBAUM, P., et al. A report: the definition and classification of cerebral palsy. Developmental medicine and child neurology. Supplement. 2007, 109(109), 8-14.

SANTOS, E.d.L.d. Correlação entre o sinal mecanomiográfico e a escala modificada de Ashworth durante avaliação clínica da espasticidade. Universidade Tecnológica Federal do Paraná: Curitiba, 2016.

YUN, S., et al. Effect of segmental muscle vibration on spasticity in children with cerebral palsy: a randomized cross-over experiment. Physiotherapy. 2015, 101(1), 700-701. https://doi.org/10.1016/j.physio.2015.03.3550

ZANINI, G., CEMIN, N.F. and PERALLES, S.N. Cerebral palsy: causes and prevalences. Fisioterapia em Movimento. 2009, 22(3), 375-381.

Received: 6 April 2020 | Accepted: 29 July 2020 | Published: 28 October 2021

This is an Open Access article distributed under the terms of the Creative Commons Attribution License, which permits unrestricted use, distribution, and reproduction in any medium, provided the original work is properly cited. 